Memorias del VII Encuentro Nacional de Experiencias en la Enseñanza de la Biología y la Educación Ambiental y II Congreso Nacional de Investigación en la Enseñanza de La Biología

\title{
IMPLEMENTACIÓN DE TALLERES SOBRE LA TEMÁTICA EXTINCIÓN DE ORGANISMOS A PARTIR DEL DIÁLOGO ENTRE RELIGIÓN Y CIENCIA
}

\section{IMPLEMENTATION OF WORKSHOPS ON THE THEMATIC EXTINCTION OF ORGANISMS FROM THROUGH DIALOGUE BETWEEN RELIGION AND SCIENCE}

\author{
William Briñez ${ }^{1}$ \\ Tatiana Romero ${ }^{2}$ \\ Juana Suarez ${ }^{3}$
}

\section{Resumen}

La clase de Ciencias Naturales es un espacio ideal para el diálogo, en el que los participantes pueden expresar su discurso, contrastarlo con el de los demás y generar conclusiones sobre una temática. En esta intervención se realizó un acercamiento al tópico extinción de organismos a partir del diálogo entre la religión y la ciencia en una institución confesional con estudiantes de primaria; durante los talleres se abordaron tres temas principales: biodiversidad, extinción y conservación, en los cuales se analizó la desaparición de diversos organismos y cómo esta puede estar influenciada por prácticas culturales tanto positivas en cuanto a la conservación, como negativas en cuanto a la extinción y pérdida de la biodiversidad. En los resultados se evidencia el desconocimiento hacia la actividad científica, lo cual lleva a que se tenga un escaso criterio de fenómenos como la extinción y se aprecien los organismos por características externas más no por su valor ecosistémico.Finalmente, es de considera que en un principio la relación ciencia-religión fue controvertida para los participantes, pero con el transcurrir de los talleres se logró la creación de un espacio de diálogo, permitiendo la generación de un conocimiento plural que puede contribuir a la solución de diversas problemáticas.

Palabras Claves: Enseñanza en ciencias, Biodiversidad, conservación.

\section{Abstract}

Science class is an ideal space for dialogue, in which participants can express their speech, contrasting it with that of others and generate conclusions about a subject. This intervention was an approach to the topic organisms dying from the dialogue between religion and science on a religious institution with elementary students;

\footnotetext{
1 Estudiante de Lic. En Biología Universidad Distrital Francisco José de Caldas. wialbrisi29@gmail.com 2 Estudiante de Lic. En Biología Universidad Distrital Francisco José de Caldas. tatiud03@hotmail.com ${ }^{3}$ Estudiante de Lic. En Biología Universidad Distrital Francisco José de Caldas. jgsuarezr@correoudistrital.edu.co
} 
Memorias del VII Encuentro Nacional de Experiencias en la Enseñanza de la Biología y la Educación Ambiental y II Congreso Nacional de Investigación en la Enseñanza de la Biología

during workshops addressed three main issues: biodiversity, extinction and conservation, in which analyzed the disappearance of various organisms and how this can be influenced by both positive cultural practices regarding conservation, as negative for extinction and biodiversity loss. In the results evidence to disregard scientific activity, which leads to a poor criterion have phenomena like extinction and are appreciated by agencies external characteristics but not by their value ecosystem. Finally, it is considered that at first the relationship between science and religion has been controversial for participants, but with the passing of the workshops was able to create a space for dialogue, allowing the generation of plural knowledge that can contribute to the solution of various problems.

Keywords: Teaching science, Biodiversity, Conservation.

\section{Introducción}

El aula de Ciencias Naturales al discutir temas fundamentales para el ser humano como el origen de las cosas, la vida y la muerte, genera conflicto en el conocimiento de los participantes, ya que enfrenta saberes y explicaciones primordiales para cada quién; es por esto, que se hace necesario promover el diálogo entre los participantes, en el que cada uno tenga respeto y valore tanto sus propias ideas como las de los demás. Lo anterior, es notorio cuando el aula de ciencias se encuentra en una institución con una orientación religiosa, ya que la relación entre Ciencia y religión como lo indica Azevedo (2011), es controvertida al acercarse cada una a la realidad de manera diferente; además, la visión de oposición entre estos dos estamentos es predominante y la escaza formación docente en este campo conllevan a que se impida un diálogo que involucre a estos dos estamentos en el aula.

Esta relación de oposición es evidente durante nuestra intervención en una institución confesional, debido a la fragmentación entre el contexto religioso en el que se encuentran las estudiantes y el de ciencia que se enseña, de manera que ambos tipos de formación eran llevadas a cabo en espacios diferentes para evitar su confrontación, conllevándonos a problematizar a las estudiantes, en búsqueda de promover el diálogo y contribuir a su formación frente a la temática de extinción.

\section{Referente Teórico: Paradigmas Epistemológicos frente a la relación Ciencia- Religión.}

Sepúlveda \& El- Hani (2004), postulan cuatro posturasalrededor de la relación ciencia-religión; (1) conflicto: Establece que la educación religiosa es incompatible con la enseñanza de las ciencias, debido a la contradicción de las doctrinas evita cualquier contacto entre estas y mucho menos en un recinto como el "aula" 
Memorias del VII Encuentro Nacional de Experiencias en la Enseñanza de la Biología y la Educación Ambiental y II Congreso Nacional de Investigación en la Enseñanza de la Biología

(Mahne y Bunge, 1996) Citado por (Sepulveda\& El-Hani, 2004).(2) independencia: la educación religiosa y la educación científica son independientes y complementarias, ya que cada una de ellas le da respuestas diferentes a las necesidades humanas (Woolnough, 1996) Citado por (Sepulveda\& El-Hani, 2004). (3) diálogo: entre la ciencia y la religión se consideran ciertas semejanzas, tales como el uso similar de modelos y analogías que buscan dar respuestas a las incógnitas de la humanidad. (4) integración: hace referencia a la creación de un campo interdisciplinario que reúna la teología y la ciencia; pasando de las posibles relaciones de los dos conocimientos a una unificación de estos (Bielfeld 1999) Citado por (Sepulveda\& El-Hani, 2004).

De igual manera, Barbour (2004) ha realizado una clasificación similar partiendo desde la postura que un individuo pueda tomar en el momento de abordar la relación ciencia-religión: En primer lugar se plantea el conflicto; debido a la rivalidad existente entre evolucionistas y cristianos, al no encontrar similitudes entre sus teorías; la segunda postura resalta la independencia, fundamentada en que ambas áreas son diferentes y responden a distintas preguntas; la tercer postura hace referencia al diálogo, desde la igualdad y respeto por ambas posturas de manera tal que cada área se dedique a su objeto de estudio sin entrar en el área de discusión de la otra y por último, se habla de la integración real de la religión y la ciencia, convocando a una unificación de las dos disciplinas (Barbour, 2004) Citado por (Azevedo, 2011).

A pesar de este conflicto en la relación entre ciencia y religión, existen pensadores tales como Matthew (1994), que proponen una educación desde una visión holística del conocimiento, tomando como referencia los diferentes saberes (cotidiano, científico, religioso, entre otros.), que permitan al individuo comprender de una mejor manera su entorno (Matthew, 1994.) Citado por (Sepulveda\& ElHani, 2004).

\section{Metodología}

El desarrollo de nuestra práctica es desde un paradigma cualitativo, bajo un enfoque descriptivo que se aborda en tres momentos:

I. Observación y caracterización: Atendiendo a la necesidad de conocer sus dificultades, sus inquietudes, sus sueños y sus gustos; se realizó la observación de las clases teniéndose un diario de registro y una grabación de audio de las mismas; como una primera actividad de reconocimiento e integración de los estudiantes se propuso una presentación colectiva por medio de un juego con un balón el cual permitía que cada estudiante hablase de sí mismo; además de esto se realizó una técnica de investigación cualitativa propuesta por Francisco Cajiao conocida como "El juego de la estrella de cinco picos"(Ballén et. Al. 2007), la cual 
Bio-grafia Escritos sobre la Biologia y su Enseñanza.

Edición Extra-Ordinaria. ISSN 2027-1034 P.p $676-684$

Memorias del VII Encuentro Nacional de Experiencias en la Enseñanza de la Biología y la Educación Ambiental y II Congreso Nacional de Investigación en la Enseñanza de la Biología

adaptamos presentándose en cada pico una pregunta que era de interés conocer tales como: ¿Qué dificultad has encontrado en la clase de Ciencias Naturales? y si fueran científicas qué tema les gustaría investigar ¿Por qué?

II. Detección del tema: A partir de las características de la población, de sus intereses y necesidades denotamos trabajar el tópico de extinción, en donde se destaca durante el proceso de observación el encuentro con algunos animales tales como: Arañas, babosas y una serpiente, los cuales provocaban cierto miedo y el deseo de matarlas, por lo que se hace necesario trabajar la temática de extinción a partir de aspectos científicos y religiosos debido al contexto en donde se encontraban.

III. Elaboración e Intervención: A partir de la relación ciencia-religión para el trabajo del tópico de Extinción con un grupo de 15 estudiantes en el grado tercero de primaria se organizaron seis actividades orientadas en el diálogo: (1) Explorando nuestro medio; (2) Preguntas de Justin acerca de Biodiversidad; (3) Profundizando sobre Biodiversidad; (4) El loro orejiamarillo y su relación con festividades religiosas, (5)Noé y la conservación de organismos y (6) Buenos y malos... ¡he ahí el dilema!

\section{Resultados}

\section{Caracterizar los estudiantes presentes en el aula de Ciencias Naturales de una Institución Educativa Confesional.}

En el desarrollo de la caracterización de las 15 estudiantes de la Institución Educativa confesional, encontramos que la percepción tanto de la ciencia como de científico, está determinada como una verdad absoluta, realizada por otros, en donde la investigación se comprende como un conjunto de elementos sin conexiones, por ejemplo a las preguntas sobre ¿Qué materiales o herramientas (libros, laboratorios, etc.) usan en la clase de Ciencias Naturales?, dijeron lo siguiente: " el tablero y el cuaderno", "libros", "nos ponen una guía así, o un libro para leer" "o nos dictan"; Así también como a la pregunta ¿Qué hace un científico?: "ah yo sé. Que está investigando materias o algo así, rocas", "va por todos los planetas", "no sé", "construye, crea las casas", "son como raros, todos", " sí que se visten de blanco, y tienen un casco".

Durante esta etapa, se evidencio la aparición de una pequeña serpiente debajo de una de las rocas, generando actitudes de miedo y la predisposición común de darle muerte, lo que conllevo a evidenciar este temor presente en las estudiantes hacia este organismo debido al desconocimiento, la imposibilidad de control. Lo anterior, permitió evidenciar la temática de la extinción dentro de la relación ciencia-religióndesde el diálogo. 
Memorias del VII Encuentro Nacional de Experiencias en la Enseñanza de la Biología y la Educación Ambiental y II Congreso Nacional de Investigación en la Enseñanza de la Biología

Por otro lado, entre las características que podemos encontrar en el PEl están enfocadas sobre tres ejes: (1) Formación en valores, orientada bajo el desarrollo integral del individuo; (2) Educación religiosa, "fomentada como la formación humana y espiritual fundamentada en la caridad, la vida eucarística, la vocación al servicio y el amor a María" y (3) Formación critica, planteada como el labor de la escuela para crear las condiciones que garanticen elaprendizaje y el desarrollo de personas autónomas, afectivas, creativas y responsables.Por tal razón, surge la necesidad de hacer una intervención en el aula de ciencias naturales en donde se tiene en cuenta el contexto religioso en el cual están inmersas las estudiantes, valiéndose de este para la enseñanza de las ciencias a partir de la instancia de diálogo que es propuesta por Sepulveda\& El-Hani, 2004.

\section{Talleres de reconocimiento: Organismos en extinción.}

Durante la realización de los talleres se resaltaron la aparición de varias actitudes por parte de las estudiantes de la Institución Educativa, partiendo de la actividad "explorando nuestro medio" se llevaron serpientes del museo de la Universidad entre otros animales, con el fin de acercarlas a sus características morfológicas y de comportamiento a partir de su contacto directo, de forma que se relacionaran con las mismas de manera diferente disminuyendo actitudes negativas, como gritos y repulsión, para favorecer la conservación de estos organismos.

Así mismo en la actividad "El loro orejiamarillo y su relación con festividades religiosas", en donde se problematizo la extinción como una consecuencia de las acciones humanas, teniendo en cuenta para esto la visión de dominación bíblica presente en génesis 1:26 "'Hagamos al hombre a nuestra imagen, conforme a nuestra semejanza; y que domine los peces del mar, las aves del cielo, el ganado y todo animal que anda sobre la tierra". A partir de esto se encontraron dos percepciones en las niñas frente a la dominación: una antropocéntrica, ya que evidencia al hombre como dominador de la naturaleza; "Yo creo que lo que lees es verdad porque dios le dijo a Dios hagamos el hombre para que se alimenten de alimento que el hombre les da." y la otra es una visión de preservación de la misma; "Que los hombres Dios los creo para que cuidáramos la naturaleza mas no la arrancáramos ni arrancáramos las plantas."

Ahondando en esta actividad algunas de ellas relacionaron la extinción como una consecuencia de la acción del hombre y de sus prácticas religiosas: "Que cortan palmas y cortan las plantas para llevar a la iglesia"; "El loro se está acabando por que los hombres cortan la palma de cera y el palo como es de madera entoncesellos lo cogen para hacer chozas, sillas, y también se acabó porque la gente para sus celebraciones religiosas como el domingo de ramos". 
Memorias del VII Encuentro Nacional de Experiencias en la Enseñanza de la Biología y la Educación Ambiental y II Congreso Nacional de Investigación en la Enseñanza de la Biología

Este acercamiento a la temática de extinción, contribuyo a romper con esas actitudes de miedo, dominación y creencias existentes en la población que pueden ser causa de la extinción de múltiples organismos, donde también se busca restablecer esa relación de equilibrio y de amor para con la naturaleza. Cabe resaltar que este trabajo se realizó con organismos endémicos, donde se abarcaron microorganismos, plantas, animales y el hombre a través de sus diversas interrelaciones que pueden contribuir a la extinción o a la preservación de los mismos.

Finalmente, durante la actividad "Noé y la conservación de organismos",en el cual trabajamos el tema de conservación simbolizando la preservación con el arca de Noé, de manera que las niñas propusieran alternativas para la conservación de organismos, y en la que ellas problematizaron sobre qué organismos deberían subir al arca, juzgando a partir de sus concepciones los organismos como buenos y malos. Evidenciándose en los siguientes apartes:"Los leones son malos porque dicen no sé, porque se comen a las personas"; “ ¿Una mariposa es buena o no? "; " el león, el león es malo"; " una culebra que son tan grandes".

\section{Análisis}

\section{En búsqueda de la relación entre educación científica y educación religiosa en pro de una formación holística.}

La enseñanza de la Ciencia suele realizarse de forma fragmentada impidiendo que el estudiante desarrolle un espíritu crítico a través del análisis de los diferentes discursos, ya que generalmente se observa a la Ciencia como un estamento "sagrado" con el que no se puede discutir ya que tiene la última verdad para todo, de manera similar a el papel que la religión llegó a tomar en un momento dado frente a la validación del conocimiento; como lo señala Giordan: "la educación científica actual es una enseñanza en la que muy raramente se intenta desarrollarel espíritu crítico, es una enseñanza donde se obliga a creer; se enseña por ejemplo la teoría de la evolución como si fuera catecismo"(Giordan, 1993).

La visión de superioridad del conocimiento científico y la escasa formación crítica de los estudiantes en este campo, se puedo evidenciar en la caracterización que se realizó a las estudiantes, donde se evidenciael desconocimiento de la actividad científica, el tradicionalismo escolar en la enseñanza de las Ciencias y la desconfianza del propio conocimiento, al buscar validarlo frecuentemente por lo recibido textualmente de la voz del profesor.

Por otro lado, VivianeCândido propone que en un mundo diverso se debe poder convivir y dialogar con diversas instancias del conocimiento tal como el científico y 
Memorias del VII Encuentro Nacional de Experiencias en la Enseñanza de la Biología y la Educación Ambiental y II Congreso Nacional de Investigación en la Enseñanza de la Biología

el religioso, por tal razón el campo educativo es propicio para esta relación ya que en él se busca la controversia para el crecimiento intelectual de los individuos. Siendo necesario romper con el paradigma de ciencia, el cual no permite que el estudiante intérprete a partir de sí mismo y de su entorno las diferentes formas en que se concibe el saber y la forma en que se hace ciencia(Cândido, 2008).

El diálogo de saberes en especial el saber religioso y científico fue promovido desde el inicio de la intervención, por ejemplo mediante la introducción de fragmentos bíblicos en el aula de clase y su análisis con los participantes de la misma, dónde no se introducían verdades absolutas sino que se dejaban interrogantes abiertos de los que cada uno sacaba sus propias conclusiones acerca de la temática que se estuviera tratando. Sin embargo, el planteamiento del diálogo en ocasiones tuvo dificultades al encontrarse participantes que no contemplaban la posibilidad de discutir fragmentos bíblicos puestoquelo asumían como verdades acabadas.

En este sentido, aunque el PEI plantea una formación integral con tres ejes: formación en valores, educación religiosa y formación crítica; la realidad es que predomina la formación en valores que aunque son importantes,pueden opacar otro tipo de habilidades como creatividad, autonomía y crítica, donde se tiende a homogenizar los saberes y conocimientos; lo cual es evidente en la inseguridad de los estudiantes en su accionar, así como la frecuente búsqueda de aprobaciónde su conocimientopor el docente.A partir delos resultadosseñalados con anterioridad surge la siguiente dimensiónde análisis:

\section{Clasificación De Organismos Buenos Y Malos}

En el taller "Noé y la conservación de organismos" aparece un conflicto entre los participantes sobre qué animales deben subir al arca etiquetándolos como buenos y malos, es de resaltar el señalamiento a la serpiente y al león como animales malos, por su relación con los otros animales, estas valoraciones son juicios morales establecidas por los sujetos y que se componen de tres elementos: el valor atribuible, el objeto valorado (actos o normas morales) y el sujeto que valora; el valor se atribuye a un objeto social establecido o creado por el ser humano, es por esto que un acto moral no es cualquier acto sino simplemente aquellos que por sus consecuencias afecten a otros, teniendo así un carácter concreto históricosocial. Siendo importante resaltar que los objetos valorados son propiamente humanos y por tanto los seres inanimados o los actos animales, no pueden ser objeto de una valoración moral (Sánchez, 1999).

De tal manera, surge la última actividad de "Buenos y malos... ¡he ahí el dilema!", porque los animales no deben ser objeto de este tipo de valoración puesto que los objetos valorados son propiamente humanos al estar situados dentro de unos 
Memorias del VII Encuentro Nacional de Experiencias en la Enseñanza de la Biología y la Educación Ambiental y II Congreso Nacional de Investigación en la Enseñanza de la Biología

principios y valores ideológicos, en esta actividad se encontraron que las apreciaciones de "malo" se producen por: peligro, convivencia con otros organismos, y la influencia de creencias religiosas en estos juicios.

En este sentido las creencias han permeado al ser humano, designando como bueno y malo a ciertos organismos, teniéndose una valoración no simplemente lógica sino histórica o real; de esta forma, lo bueno durante la edad media provenía de la voluntad divina y lo malo, de lo diabólico o que le contradice (Sánchez, 1999); pero en la naturaleza no existe tal connotación porque los animales cumplen un papel en el equilibrio de la naturaleza y su inexistencia es una perdida invaluable no solo para el ecosistema, sino para el hombre.

\section{Conclusiones}

El abordaje del tópico de extinción desde la relación entre Ciencia y religión, fue tomada en un inicio por las estudiantes desde una postura de controversia, que al pasar las diferentes actividades, el aula se fue transformando en un espacio de diálogo, en el que se respectaba, se debatía y se compartían saberes. Resaltando la importancia de seguir acercándonos al aula de ciencia con temáticas similares, que permitan al estudiante tomar diferentes conocimientos de su entorno para darle solución a los dilemas que se presenten en su cotidianidad.

Finalmente, la relación entre Ciencia y religión es una temática importante, que se debe seguir trabajando desde los diferentes tópicos presentes en el aula de ciencias, permitiéndoles a los estudiantes acercarse a debates de diferente índole.

\section{Bibliografía}

- Azevedo, H. (2011). Competência comunicativa de futuros professores frente à diversidade religiosa na abordagem do tema "origens do universo". Universidade estadual paulista "julio de mesquita filho"- programa de pós graduação em educação para a ciência., Bauru.

- Ballén, M., Pulido, R., \&Züñiga, S. (2007). Abordaje hermenéutico de la investigación cualitativa Teorías, proceso, técnicas. Bogotá: Universidad Cooperativa de Colombia.

- Cândido, V. (2008). Epistemologia da controversia para o ensino religioso: aprendendo e ensinando na diferencia fundamentos no pensamiento de Franz Rosenzweig. Sau Paulo: Pontificia Universidade católica de saopaulo PUC-SP.

- Giordan, A. (1993). La enseñanza de las Ciencias. España: Siglo XXI. 
Bio-grafia Escritos sobre la Biologia y su Enseñanza.

Edición Extra-Ordinaria. ISSN 2027-1034 P. p 676-684

Memorias del VII Encuentro Nacional de Experiencias en la Enseñanza de la

Biología y la Educación Ambiental y II Congreso Nacional de Investigación en la Enseñanza de la Biología

- Sánchez, A. (1999). Ética. Barcelona: Crítica S.A.

- Sepulveda, C., \& El-Hani, C. (2004). Quando visões de mundo se encontram: Religião e ciência na trajetória de formação de alunos protestantes de uma licenciatura em ciências biológicas. InvestigaçõesemEnsino de Ciências, 137-175. 